\title{
Validity of the Draw a Person: A Quantitative Scoring System (DAP:QSS) for Clinically Evaluating Intelligence
}

\author{
Alda Troncone $^{1}\left[\right.$ Antonietta Chianese $^{1} \cdot$ Alfonso Di Leva ${ }^{1} \cdot$ Maddalena Grasso $^{1} \cdot$ Crescenzo Cascella $^{1}$
}

Accepted: 4 September 2020 / Published online: 12 September 2020

(c) The Author(s) 2020

\begin{abstract}
To assess the psychometric properties of the Draw a Person: A Quantitative Scoring System (DAP:QSS), in 2543 children $(M=11.43 \pm 3.06$ years), correlations between drawings scores and Raven's Matrices scores, age, and academic achievement were examined. Although older children ( $>11$ years) obtained higher drawing scores than younger ones $(p<0.001)$, age significantly correlated with DAP:QSS scores only in children younger than 11 years $(r=0.493, p<0.001)$, indicating conflictive evidence for construct validity and a possible ceiling effect. No correlations emerged between DAP:QSS scores and grades $(r=0.056, p=0.097)$. DAP:QSS scores were significantly associated with Raven's Matrices score, but low correlation coefficients $(0.156-0.498)$, low sensitivity (0.12), and high false negative (87.9\%) and positive (82\%) rates suggest poor DAP:QSS validity as an intelligence measure. The researchers concluded that DAP:QSS failed to produce a psychometrically sound assessment of children's intellectual functioning.
\end{abstract}

Keywords Intelligence · Draw a Person: A Quantitative Scoring System · Children · Adolescents · Scholastic achievement

The use and usefulness of human figure drawing tests as measures of intellectual ability have generated considerable debate [1-3]. Among the several methods of scoring human figure drawings, the Draw a Person: A Quantitative Scoring System (DAP:QSS) was developed by Naglieri in 1988 as an updated means of scoring the classic draw-a-person test. The DAP:QSS was designed for use with 5- to 17-year-olds as a nonverbal measure not influenced by linguistic variables that "can provide reliable information about intellectual status to supplement other intelligence test" [4, p. 2]. Created with reference to a well-normed standardization sample, the DAP:QSS uses three stimuli (i.e., drawings of a man, a woman, and the self) devised to avoid the possible confounding variables of fashion and dress.

Evidence suggests that the DAP:QSS generally yields high coefficients for intra- and inter-rater reliability [4-7], internal consistency, and test-retest reliability [4]. By contrast, the validity of the DAP:QSS as an instrument for assessing intelligence has yet to be satisfactorily demonstrated.

Alda Troncone

alda.troncone@unicampania.it

1 Department of Psychology, University of Campania "Luigi Vanvitelli", Viale Ellittico 31, Caserta, Italy
Moderate-to-high correlations with measures of verbal intelligence (e.g., Wechsler scales) $[5,8,9]$ and nonverbal intelligence [4, 10-12], even among children and adolescents with learning disabilities [13], suggest that Naglieri's DAP:QSS test can serve as a general screening measure of children's and adolescents' intelligence. Naglieri's individual DAP drawings have also shown good correlations with the Goodenough-Harris's drawing test $[14,15]$.

However, other researchers have found little support for the validity of the DAP:QSS as a tool for evaluating children's intellectual ability and assessing children with mental disabilities [3, 16, 17]. Indeed, some researchers have not only concluded that the DAP:QSS is not a valid measure of intellectual ability and should not be used as a screening tool [7], but also suggested that human figure drawing tests should be eliminated from the repertoire of psychological assessment tools [18].

In examination of the utility of any measure of intelligence, relevant importance has been given to its ability to predict scholastic performance [3, 19]; consequently, the association between DAP:QSS and measures of academic achievement has also been examined, the results of which provide even more conflicting evidence. In particular, whereas some researchers found that the DAP:QSS cannot predict academic performance [3,12, 17], others have 
described significant correlations between the DAP:QSS and scholastic achievement $[4,10,20]$.

In recent investigations of the strengths and weaknesses of the DAP:QSS, researchers have analyzed whether DAP:QSS scores can be improved with practice [21], can be useful to identify highly gifted children [22], and are vulnerable to deliberate distortion by adolescents and young adults [23], due to generational changes (i.e., Flynn effect) [24], or due to the effects of gender [25]. In other work, Gentle, Powell, and Sharman [26] have examining the use of DAP:QSS as a protective exercise that lessens the impact of biased questions upon child witnesses. The DAP:QSS was also found to be specifically useful for evaluating cognitive and motor deficits of exceptionally preterm children [27].

Except for two studies that referred to an original normative sample of 2622 children aged 5-17 years in the United States [4, 28], studies geared toward analyzing the psychometric properties of the DAP:QSS have been conducted mostly with small samples of children with narrow age ranges in the United States ([5] $N=200,6-15$-yearolds; [7] $N=51,6-16$-year-olds), Canada ([11] $N=598$, 6-10-year-olds), Greece ([14] $N=114$ elementary-school children), Lithuania ([16] $N=165,6-16$-year-olds), Japan ([12] $N=400,6-12$-year-olds), New Zealand ([7] $N=125$, 5-6-year-olds), and Italy ([15] $N=184$ elementary-school children). Despite those studies, the psychometric properties of the DAP:QSS and its relationship with other commonly used measures of nonverbal ability need to be elucidated. As Abell et al. [5] have stated, "If they are to have any clinical validity, drawing tests must be examined with different population of children and different standard intelligence tests" [5, p. 206].

To address that need, the study presented here was conducted with a large sample of Italian children across a broad age range, with the aim of clarifying the psychometric properties of the DAP:QSS, especially in terms of its construct validity, concurrent validity, and usefulness in screening for intellectual difficulties. In particular, because construct validity can be determined by differentiating ability according to the child's age $[4,29]$, the general developmental trend in DAP:QSS scores was analyzed as well. By contrast, concurrent validity was examined by comparing the DAP:QSS with more standard measures of nonverbal intelligence (i.e., the Raven Progressive Matrices).

Additionally, in order to simultaneously determine other aspects of the potential usefulness of the DAP:QSS, the relationship between children's drawings and their academic achievement was examined for criterion-related validity by analyzing correlations between DAP:QSS scores and academic grades. Last, the usefulness of the DAP:QSS as screening tool for intellectual ability was investigated by verifying its ability to accurately identify children classified as having mental disabilities according to a valid measure of intelligence. Reliability tests-specifically, inter-rater and internal consistency coefficients calculation-were also conducted.

Specifically, the following four research questions were addressed.

1. What is the construct validity of the DAP:QSS?

2. What is the concurrent validity of the DAP:QSS and Raven's Matrices with a group of individuals in the school-aged population?

3. What correlations exist between DAP:QSS and grades, and is it possible to predict children's school achievement based on their DAP:QSS scores?

4. Is the DAP:QSS capable of discerning children who might be at risk for intellectual difficulties?

In examining those relationships, given evidence suggesting the significant influence of socioeconomic status (SES) on drawing performance [19], intelligence tests [30], and academic achievement [31, 32], SES was also taken into account. Similarly, in line with data describing gender differences in Naglieri's drawings [12, 25, 27], boys' and girls' drawings were compared.

In accordance with the Naglieri's assumption that DAP:QSS is a nonverbal intelligence measure [4], and with previous evidence supporting an association between DAP:QSS and measures of intelligence $[4,5,8-13,15]$ and scholastic achievement $[4,10,20]$, this study hypothesizes that the Naglieri scoring system yields values that can be related to age, Progressive Matrices scores, and gradesbut modestly, and not to the same extent that a more complex measure of IQ will. Similarly, given the poor evidence supporting DAP:QSS as an intelligence screening device $[7,17]$, it is expected that Naglieri's drawings will show low accuracy in identifying children with low intellectual functioning.

\section{Method}

\section{Participants}

The study was conducted using a convenience sample of school-age children from local public primary, secondary, and high schools in five cities in Campania, Italy, who volunteered to participate. The schools were selected both on the basis of accessibility and to obtain roughly equal numbers of students from elementary, middle, and high school. A parent or guardian of each child who agreed to participate was asked to complete and sign a consent form. Students aged from 5 to 17 years who submitted signed parental permission forms indicating their consent to participate were included in the sample. Participants who needed an assistant teacher 
for a mental or physical disabilities were excluded, because due to the level, severity, and features of their disabilities, they would have probably requested specific adjustments in measurement procedures that were not entirely compatible with the assessment method or that would even negatively impact test administration (due to physical inaccessibility to rooms dedicated to evaluations, difficulties in moving, requiring presence of a special needs teacher, etc.). No extra credit was given for participation.

\section{Procedure}

From October 2011 to October 2014, evaluations were made anonymously and collectively, with four children at a time distanced from each other to prevent peer influence, during class time in a room made available by the school and in the absence of the teacher. Participants were not told about the purpose of the study. Following the distribution of the normative sample age for Raven's Colored Progressive Matrices (RCPM) [33], children aged from 5 to 11 years were evaluated with RCPM, whereas ones older than 11 years were evaluated with Raven's Standard Progressive Matrices (RSPM). Both the RCPM and RSPM were administered without any time limit.

The students' grades, when available, were obtained by directly consulting the students' report cards. Academic achievement was evaluated as a mean of the grades obtained in six core subjects (i.e., Italian, history, geography, English, math, and science).

Parents were asked to complete a questionnaire addressing SES upon signing the consent form.

Four psychologists with master's degrees adequately trained in the relevant techniques administered and scored all tests with reference to scoring instructions and the classification of intelligence included in the examiner's manual for the DAP:QSS [4], RCPM and RSPM [33, 34]. The scoring of drawings was made without prior knowledge of the scores of the same subject for the RCPM or RSPM. The study was approved by the local ethics committee.

\section{Measures}

\section{Draw-a-Person: A Quantitative Scoring System (DAP:QSS)}

The DAP:QSS [4] was developed to measure nonverbal aspects of intelligence in children 5-17 years old. Children are required to draw a picture of a man, a woman, and themselves. With an administration time of 5 min per drawing, the instrument can be administered individually or in a group. The system requires the application of the same 64 items to rate all three drawings on the basis of 14 scoring criteria: arms, attachment of limbs, clothing, ears, eyes, feet, fingers, hair, head, legs, mouth, neck, nose, and torso. Points are awarded for the inclusion of various body parts, the elaboration of the parts, their attachment to each other, their individual and total proportionality, and their location in the drawing. Three separate raw scores from 0 to 64 for all three drawings and a total score can be computed. Total scores can be converted to standard scores and percentile ranks or age equivalents.

The DAP:QSS was normed with a stratified sample of 2622 children 5-17 years old who were representative of 1980 U.S. Census data. The normative sample was stratified for age, sex, race, geographic region, ethnic group, socioeconomic status, and community size. Good psychometric properties such as reliability (i.e., internal reliability coefficients from 0.83 to 0.89 for the total and from 0.56 to 0.78 for the man, woman, and self drawings) and the construct and concurrent validity (i.e., significant correlations with the other measure of nonverbal ability and achievement in reading and math) are reported in the manual for the DAP:QSS [4].

\section{Raven's Progressive Matrices}

Described as "the paradigm test of nonverbal, abstract reasoning ability" [35, p. 564], Raven's Progressive Matrices are the oldest, most widely used tests for nonverbal intelligence $[36,37]$. They consist of a series of multiple-choice items concerning abstract reasoning of increasing difficulty. Each item presents a logical pattern with a missing element. For each item, participants are asked to identify the correct element from six or eight cells provided below the figure that would best complete the pattern. A participant's score is the number of correct answers.

In particular, Raven's Colored Progressive Matrices (RCPM) [38] was designed for individuals in the developmental stage (i.e., 3-12 years of age). It includes 36 items, all colored to attract and maintain children's attention, divided into three subtests of 12 items each. The maximum possible score for the RCPM is 36. The related Raven's Standard Progressive Matrices (RSPM) [39] was designed for older children and adults. It includes 60 items in five sets (i.e., A-E), each containing 12 items. The maximum possible score for the RSPM is 60. The RSPM and the RCPM have been regularly evaluated for reliability and validity in various countries all over the world, and they have been shown to be a valid measure of nonverbal cognitive ability [40-44].

To evaluate participants' performance, the raw scores were compared to recent normative scores (i.e., average scores for age groups and relative centiles), collected during the latest Italian standardization of the RCPM and the RSPM [33, 34]. 


\section{Barratt Simplified Measure of Social Status (BSMSS)}

The BSMSS [45] is a measure of SES based upon the widely used Hollingshead Four Factor Measure [46] with updated job categories. It provides a simple measure of SES based on marital status, current employment status (or former status for retirees), level of education, and occupational prestige. For school-age individuals, that index is computed as a combination of parents' educational level and work activity. Occupation was coded in nine groups ranging from 1 (farm laborers and menial workers) to 9 (executives and major professionals), whereas education was coded in seven levels ranging from 1 (less than a 7 th-grade education) to 7 (graduate degree). Level of education was adjusted to suit the Italian education system. Scores vary from 8 to 66 , and higher scores indicate higher SES.

\section{Statistical Analysis}

Cronbach's alpha $(\alpha)$ was used to assess the homogeneity of DAP:QSS scores. Inter-examiner reliability was determined by calculating the Cohen's kappa coefficient for the agreement of intelligence classification assigned by each pair of raters to the same drawings in a random sample of 300 drawings. Independent samples $t$-test was carried out in order to investigate group differences in mean scores (boys vs. girls; younger ( $\leq 11$ years old) vs. older ( $>11$ years old)).

Additionally, Pearson product-moment correlations for bivariate correlations and partial correlations removing effect of SES were conducted to assess the relationships between DAP:QSS score and age, DAP:QSS score and RCPM or RSPM score, and between the DAP:QSS scores and academic grades. Bivariate correlation coefficients were compared using the Fisher $r$-to- $z$ transformation. Stepwise multiple regression analyses were conducted to more closely evaluate how well DAP:QSS total score and SES-predicted Raven Progressive Matrices; drawings, Raven Progressive Matrices, and SES scores predicted grades.

The frequency distribution of intelligence classifications according to DAP:QSS, RCPM, and RSPM scores was computed. Because comparable standard scores for the RCPM and RSPM are unavailable, differences between DAP:QSS and RCPM or RSPM scores were assessed by comparing range percentiles with the Wilcoxon signed-rank test for a single sample. Following Willcock et al. [7], to ascertain the effectiveness of the DAP:QSS as a screener of intellectual ability, the scores of children classified as having low or very low intellectual functioning according to the RCPM or RSPM (percentile < 15.5) [33] were compared with their scores on the DAP:QSS for the false negative rate. Conversely, the scores of children identified as having borderline or deficient intellectual functioning according to the DAP:QSS (percentile $\leq 8$ ) [4] were compared with their scores on the RCPM or RSPM for the false positive rate. The concordance between drawings and Raven Progressive Matrices in classification of low intellectual functioning was calculated as sensitivity, specificity, and positive predictive values (PPV) of DAP:QSS total score (implying borderline/deficient intelligence and no borderline/deficient intelligence) and assessed against RCPM/RSPM intelligence classification (indicating low/very low intellectual functioning or no low/very low intellectual functioning). Effect sizes were calculated by means of Cohen's $d$ for $95 \%$; confidence intervals estimates for the effect sizes were also computed.

Raw scores of variables were used in all analyses, and $p$ values $<0.05$ were considered to indicate statistical significance. All statistical analyses were performed with the Statistical Package for the Social Sciences version 21.0 for Macintosh.

\section{Results}

\section{Characteristics of Participants}

Twenty-eight schools agreed to participate: nine elementary schools (i.e., four in Naples, one in Avellino, two in Caserta, one in Benevento, and one in Salerno), 11 middle schools (i.e., six in Naples, one in Avellino, two in Caserta, one in Benevento, and one in Salerno), and eight high schools (i.e., four in Naples, one in Avellino, one in Caserta, one in Benevento, and one in Salerno).

Of the 2703 parents who were approached, 2578 consented to allow their children to participate in the study, whereas 125 did not, for a participation rate of $95.37 \%$. Thirty-five parents forgot to read, sign, or return the consent form for their children, 58 children were not given permission from their parents to participate, and 32 children were absent on the day of their test. Of the 2578 participants evaluated, 35 were excluded from analysis: 31 older than 12 years who were erroneously assessed with the RCPM, two for returning incomplete tests that provided RSPM or DAP:QSS data, and two younger than 11 years who were erroneously evaluated with the RSPM. Grades were collected for 894 participants.

The final sample consisted of 2543 participants, mostly of low or middle SES, with the mean grade corresponding to C on the American grading scale. Participants' characteristics are shown in Table 1.

The reliability analysis (in terms of inter-rater agreement across the four raters) showed Cohen's kappa coefficients ranging from 0.797 to 0.99 .

The Cronbach's alpha coefficients, means, and standard deviations for each of the three drawings and DAP:QSS total score appear in Table 2, which also shows DAP:QSS scores by the gender and age of participants. 
Table 1 Participants' characteristics $(N=2543)$

\begin{tabular}{ll}
\hline Boys and girls $(n)$ & 1135 and 1408 \\
Age (years with months) & $11.43(3.06)$, range: $5.04-17.8$ \\
Socioeconomic status & $29.25(11.96)$, range: $0.0-66$ \\
Grades $(n=894)$ & $7.68(1.16)$, range: $4.58-10.0$ \\
Elementary school $(n, \%)$ & $985(38.73)$ \\
Middle school $(n, \%)$ & $854(33.58)$ \\
High school $(n, \%)$ & $704(27.69)$ \\
\hline
\end{tabular}

Data are presented as mean values with standard deviations unless stated otherwise

Girls obtained higher DAP:QSS scores than boys for all drawings $(\operatorname{man}, t(2541)=-3.620, p=0.000$, $d=-0.15$ [95\% CI for effect size: $-0.22,-0.07$ ]; woman, $t(2541)=-6.727, p=0.000, d=-0.27$ [95\% CI for effect size: $-0.35,-0.19]$; self, $t(2541)=-4.876, p=0.000$, $d=-0.2$ [95\% CI for effect size: $-0.28,-0.12]$ ), as well as total score $(t(2541)=-5.511, p=0.000, d=-0.22[95 \%$ CI for effect size: $-0.30,-0.14])$. By contrast, RCPM and RSPM mean scores did not differ between boys (RCPM: $26.38 \pm 6.34$; RSPM: $43.97 \pm 8.39$ ) and girls (RCPM: $26.11 \pm 6.23$; RSPM: $44.56 \pm 7.96)$ in the sample (RCPM $t(1037)=0.700, p=0.484$; RSPM $t(1502)=-1.368$, $p=0.172)$ (data not shown).

\section{Construct Validity of the DAP:QSS}

Children older than 11 years obtained higher raw scores than younger children for all drawings and for the total score (man, $t(2541)=-21.469, p<0.001, d=-0.85$ [95\% CI for effect size: $-0.91,-0.75]$; woman, $t(2541)=-24.844$, $p<0.001, d=-1.01$ [95\% CI for effect size: -1.09 , $-0.92]$; self, $t(2541)=-27.235, p<0.001, d=-1.1[95 \%$ CI for effect size: $-1.18,-1.01]$; total, $t(2541)=-27.002$, $p<0.001, d=-1.09$ [95\% CI for effect size: -1.17 , $-1.01]$ ) (Table 2).
As shown in Table 3, age significantly correlated with DAP:QSS scores across the sample for both boys (total, $r=0.526, p<0.0001$; man, $r=0.430, p<0.001$; woman, $r=0.483, p<0.001$; self, $r=0.555, p<0.001)$ and girls (total, $r=0.445, p<0.0001$; man, $r=0.347, p<0.001$; woman, $r=0.428, p<0.001$; self, $r=0.430, p<0.001$; data not shown).

However, in an analysis of the sample grouped by age, correlations between DAP:QSS scores and age emerged only for participants aged 11 years or younger but not older ones, even when the effect of SES was considered, as shown in Table 3.

\section{Concurrent Validity of the DAP:QSS and Its Association with Academic Achievement}

DAP:QSS scores were significantly and positively correlated with Raven Matrices scores, with $\mathrm{r}$ values ranging from 0.156 to 0.498 , even when the effect of SES was removed, with the exception of the association between DAP:QSS score and RSPM score for the drawing of a man, as shown in Table 3. Correlations between DAP:QSS total score and RCPM score were significantly higher $(z=8.643, p<0.001)$ than those between DAP:QSS total score and RSPM score (Table 3).

Across the sample, no correlations were observed between DAP:QSS scores and grades regardless of SES.

In stepwise regression analysis testing DAP:QSS scores and SES as predictors, DAP:QSS total score emerged as a significant predictor of RCPM scores by explaining approximately $24.7 \%$ of the variance $\left(R^{2}=0.247, F=338.469\right.$, $d f=1, p<0.001)$. In the second step of analysis, the addition of SES in the regression equation increased the explained variance by $1.7 \%\left(R^{2}=0.263, F=184.615, d f=2, p<0.001\right)$. In a subsequent stepwise regression analysis testing DAP:QSS scores and SES as predictors, SES surfaced as a significant predictor of RSPM scores by explaining approximately $3.9 \%$ of the variance $\left(R^{2}=0.039, F=59.356, d f=1\right.$, $p<0.001)$. In the second step of that analysis, the addition of DAP:QSS total scores in the regression equation increased

Table 2 Cronbach's alpha $(\alpha)$ coefficients and mean scores $(S D)$ for Draw a Person: A Quantitative Scoring System (DAP:QSS scores in the sample $(N=2543)$; drawings' scores compare boy and girls as well as older and younger participants

\begin{tabular}{|c|c|c|c|c|c|c|}
\hline DAP:QSS & $\alpha$ & $\begin{array}{l}\text { Total sample } \\
(N=2543) \\
M(S D)\end{array}$ & $\begin{array}{l}\text { Boys } \\
n=1135 \\
M(S D)\end{array}$ & $\begin{array}{l}\text { Girls } \\
n=1408 \\
M(S D)\end{array}$ & $\begin{array}{l}\leq 11 \text { years old } \\
n=1039 \\
M(S D)\end{array}$ & $\begin{array}{l}>11 \text { years old } \\
n=1504\end{array}$ \\
\hline Man & 0.789 & $42.44(9.7)$ & $41.65(10.26)$ & $43.07(9.19)^{* * *}$ & $38.01(7.81)$ & $45.49(9.71)^{* * *}$ \\
\hline Woman & 0.749 & $42.36(8.71)$ & $41.06(9.09)$ & $43.4(8.25)^{* * *}$ & $37.72(7.88)$ & $45.56(7.77)^{* * *}$ \\
\hline Self & 0.761 & $42.97(8.86)$ & $42.01(9.27)$ & $43.75(8.44)^{* * *}$ & $37.91(7.81)$ & $46.47(7.79)^{* * *}$ \\
\hline Total & 0.907 & $127.75(24.91)$ & $124.7(26.51)$ & $130.22(23.27) * * *$ & $113.6(22.03)$ & $137.53(21.92)^{* * *}$ \\
\hline
\end{tabular}

$* p<0.05 ; * *<0.01 ; * * * p<0.001$ 


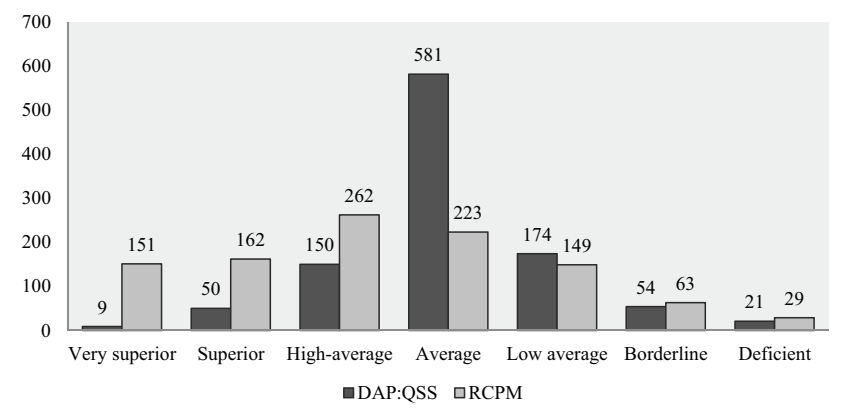

Fig. 1 Number of participants classified according to Raven's Colored Progressive Matrices (RCPM) and Draw a Person: A Quantitative Scoring System (DAP:QSS) scores and related intelligence categories

the explained variance in RSPM scores by $3.2 \%\left(R^{2}=0.071\right.$, $F=55.850, d f=2, p<0.001)$.

In another stepwise regression analysis testing DAP:QSS scores, RCPM score, and SES as predictors of grades, SES emerged as a significant predictor by explaining approximately $8.8 \%$ of the variance $\left(R^{2}=0.088, F=48.986, d f=1\right.$, $p<0.001)$. In the second step of the analysis, the addition of DAP:QSS scores in the regression equation increased the explained variance by $1.7 \%\left(R^{2}=0.105, F=29.611\right.$, $d f=2, p<0.001)$. Testing DAP:QSS scores, RSPM score, and SES as predictors of grades revealed that RSPM score was a significant predictor $\left(R^{2}=0.23, F=113.786, d f=1\right.$, $p<0.001)$, as was SES $\left(R^{2}=0.327, \Delta R=0.097, F=92.285\right.$, $d f=2, p<0.001)$ and DAP:QSS total score $\left(R^{2}=0.349\right.$, $\Delta R=0.022, F=67.603, d f=3, p<0.001)$.

\section{Screening Ability of the DAP:QSS}

The number of participants classified according to DAP:QSS and Raven Matrices scores are shown in Fig. 1 ( $\leq 11$ years participants), Fig. 2 (> 11 years participants), and Table 4 (all participants, column Total).

A comparison of the percentile ranks obtained by participants on the RCPM or RSPM and the DAP:QSS with the Wilcoxon signed-rank test revealed that scores obtained on the RCPM or RSPM differed significantly from those on the DAP:QSS (RCPM, $z=-13.109, p<0.001$; RSPM, $\mathrm{z}=-10.966, p<0.001)$.

As shown in Table 4, of the 182 participants indicated to have borderline deficient intelligence or deficient intelligence-all obtained percentile scores of 8.0 or less on the DAP:QSS-82\% ( $n=131$ of 182$)$ did not have borderline deficient or deficient intellectual functioning as measured by the RCPM or RSPM, for a high false positive rate. According RCPM and RSPM scores, of those 131 participants, 40 (30.5\%) had low average intelligence, $36(27.4 \%)$ had average intelligence, 37 (28.3\%) had high 


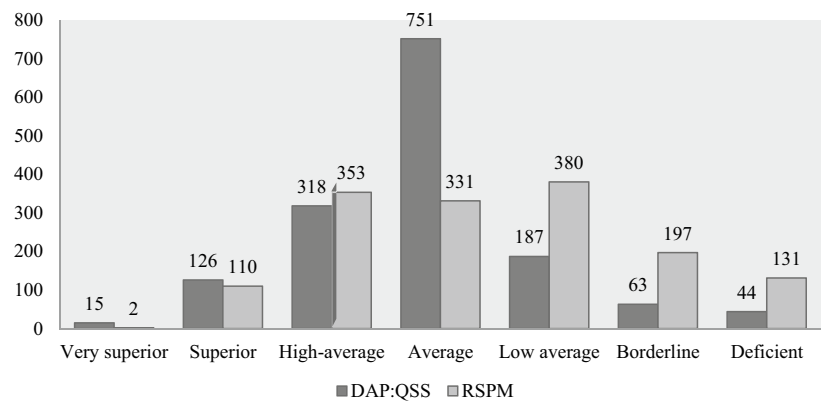

Fig. 2 Number of participants classified according to Raven's Standard Progressive Matrices (RSPM) and Draw a Person: A Quantitative Scoring System (DAP:QSS) scores and related intelligence categories

average intelligence, 14 (10.7\%) had superior intelligence, and four (3.1\%) had very superior intelligence (Table 4 ).

Of the 420 children who obtained percentile scores of 15.5 or less on the RCPM or RSPM, $87.9 \%$ ( $n=369$ of 420) were not identified as having borderline intellectual functioning according to the DAP:QSS, for a high false negative rate. The DAP:QSS erroneously evaluated those participants by classifying 75 of $369(20.3 \%)$ as having low average intelligence, $229(62.1 \%)$ as having average intelligence, 49 as having high average intelligence $(13.3 \%)$, and $16(4.3 \%)$ as having superior intelligence (Table 4).

Of the 75 children 11 years old or younger who obtained percentile scores of 8 or less on the DAP:QSS, $73.3 \%$ ( $n=55$ of 75 ) were not of borderline or deficient intellectual functioning as measured by the RCPM or RSPM, for a high false positive rate (Fig. 3, Drawings a and b). Of the 92 children older than 11 years of age who obtained percentile scores of 15.5 or less on the RCPM or RSPM, 78.3\% ( $n=72$ of 92 ) were not identified as having borderline intellectual functioning by the DAP:QSS, for another high false negative rate (Fig. 3, drawings $\mathrm{c}$ and d).
For the whole sample, the sensitivity, specificity, and PPV of the DAP:QSS for identifying low intellectual functioning were $0.12,0.94$, and 0.28 respectively.

\section{Discussion}

The study presented here was conducted with a large sample of Italian children $(N=2543)$ across a broad age range, with the aim of examining the psychometric properties of the DAP:QSS. To date, the American norms collected in a sample of 2622 individuals aged from 5 to 17 years have constituted the only available guide for scoring and interpreting Naglieri's drawings. Therefore, the results obtained provide Italian norms for the DAP:QSS as well.

The present findings support the reliability of the DAP:QSS, in terms of both internal consistency and interrater reliability. In particular, Cronbach's alpha coefficients indicated the good internal consistency for each DAP:QSS drawing, and very good internal consistency for DAP:QSS total score. The internal consistency reliabilities for the single drawings were lower than that of DAP:QSS total score and quite consistent with others reported in the test manual [4].

A significant gender-based effect was also found, which corroborates findings from Saklofske et al. [12] and Schepers et al. [27], as well as normative data. However, it should be noted that Naglieri [4] considered such an effect to be low and lacking sufficient practical significance to require different norms on the basis of gender.

In terms of construct validity, although older children obtained higher raw scores than younger ones for all drawings and for the total score, the findings indicate conflictive evidence for age differentiation: age significantly correlated with DAP:QSS scores only for children 11 years or younger, suggesting that a ceiling effect may have occurred. As affirmed by Scott [19] regarding

Table 4 False negatives $(\mathrm{N}=369)$ and positives $(\mathrm{N}=131)$ of intelligence functioning according to Draw a Person: A Quantitative Scoring System (DAP:QSS) and Raven's Colored Progressive Matrices (RCPM) or Raven's Standard Progressive Matrices (RSPM) scores

\begin{tabular}{|c|c|c|c|c|c|c|c|c|}
\hline \multirow[t]{2}{*}{ DAP:QSS score } & \multicolumn{8}{|c|}{ RCPM or RSPM score } \\
\hline & Very low & Low & Low average & Average & High average & Superior & Very superior & Total \\
\hline Deficient & 12 & 6 & 15 & 15 & 14 & 2 & 1 & 65 \\
\hline Borderline/deficient & 19 & 14 & 25 & 21 & 23 & 12 & 3 & 117 \\
\hline Low average & 33 & 42 & 82 & 80 & 73 & 30 & 21 & 361 \\
\hline Average & 78 & 151 & 279 & 292 & 325 & 131 & 76 & 1332 \\
\hline High average & 14 & 35 & 90 & 101 & 131 & 66 & 31 & 468 \\
\hline Superior & 4 & 12 & 32 & 39 & 44 & 28 & 17 & 176 \\
\hline Very superior & $\mathbf{0}$ & $\mathbf{0}$ & 6 & 6 & 5 & 3 & 4 & 24 \\
\hline Total & 160 & 260 & 529 & 554 & 615 & 272 & 153 & 2543 \\
\hline
\end{tabular}

False negatives appear in bold and false positives in italic 


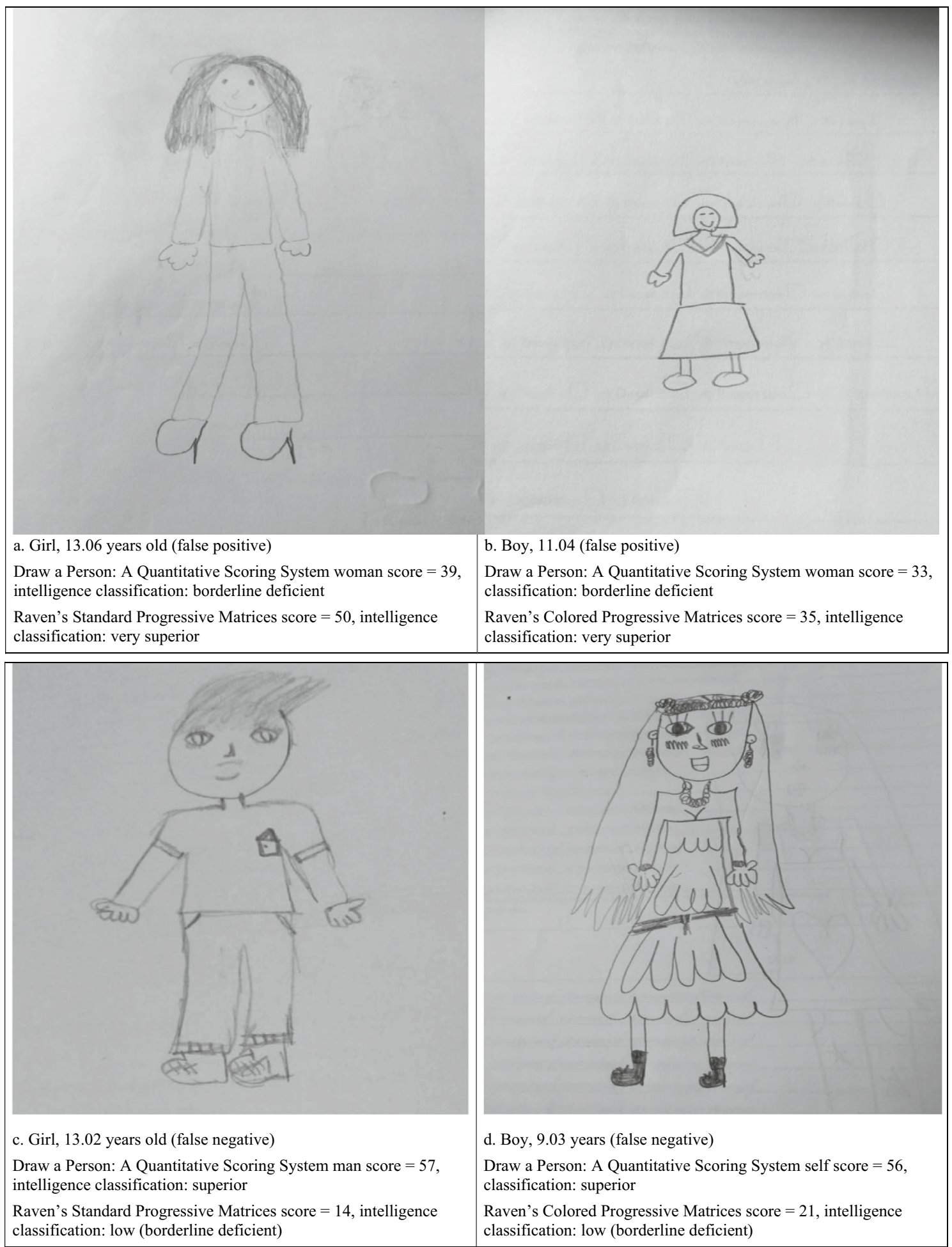

Fig. 3 Examples of false positives and negatives

Goodenough-Harris's drawing test scores and by Naglieri [4] regarding the DAP:QSS, human figure drawings differentiate performance only between age groups between 5 and 11 or 12 years old when their test scores show a substantial increment associated with increased age. Due to the test's ceiling effect, no gain in scores could be expected for children older than 12 years of age. That leveling off in scores may be due to the presence of a finite number of items in 
the drawings for which a typically developed adolescent can readily earn points. Moreover, in early adolescence, the transition from habits of concrete to abstract conceptualization favors increasing distance from material realities and concrete details [19]. Ultimately, the irregularly-changing developmental trend in the drawings' scores that was found in the present investigation provides inconsistent DAP:QSS construct validity data.

In terms of concurrent validity, the findings indicate positive and significant-albeit modest-correlations between children's scores on the DAP:QSS and their scores on standard measures of nonverbal intelligence (i.e., RSPM and RCPM) regardless of the effect of SES. In particular, correlation coefficients ranging from 0.156 to 0.498 were comparable and sometimes higher than the correlation levels between the DAP:QSS and analogous measures of nonverbal intelligence (i.e., Matrix Analogies Test-Short Form and RCPM), as reported by previous studies ([4] $r=0.19-0.31$; [10] $r=0.32$; [11] $r=0.30-0.37$; [12] $r=0.35-0.50$; [13] $r=0.35-0.50 ;$ [15] $r=0.35-0.44)$. Accordingly, a small amount of variance in a child's Raven's Matrices scores is predicted by their drawings.

The associations between DAP:QSS and RCPM scores were significantly stronger than the associations between DAP:QSS and RSPM scores. Because the interpretation and features of children's drawings are confounded by maturational conditions [47], it can be hypothesized that the influence of confounding variables, including artistic ability, low motivation, personal interest in drawing, the degree of adolescent adherence to test instructions, and emotional difficulties frequently experienced during adolescence, could have been played a role in the weak association between adolescents' drawings and their RSPM performance.

However, as highlighted by Gresham [18], "it does not make much psychometric sense to use human figure drawings to validate the results of an intelligence test when the correlation between the two is between 0 and 0.4 , whereas the correlations among intelligence tests is between 0.8 and 0.9 " (p. 183). With this in mind, the overall correlations between drawings and Raven matrices described here are substantially low, indicating that the DAP:QSS does not contribute incrementally valid information to intelligence test results.

The DAP:QSS did not correlate significantly with academic achievement and demonstrated very little usefulness in predicting such achievement. Unlike findings presented in the test manual [4] indicating DAP scores correlated significantly with reading and math achievement among students in Grades 4-12 and in other studies [10, 15, 20], those results suggest that a limited relationship exists between DAP:QSS and grades. Such results should be considered to take into account that academic achievement is a product of the dynamic interaction of a hierarchy of factors
[48, 49] such that grades (e.g., teachers' evaluations) may not always accurately reflect general cognitive abilities at the individual level. Even though the results should be interpreted with caution, they should also be recognized as further evidence highlighting that the DAP:QSS is flawed in predicting scholastic performance.

Concerning the usefulness of administering the DAP:QSS to identify children possible at risk of intellectual difficulties, the DAP:QSS was not as effective in screening intellectual ability. Low sensitivity, along with high false positive and negative rates that were quite similar between participants younger and older than 11 years, suggest that the DAP:QSS failed to identify numerous children with intellectual difficulties and falsely identified children with normal and even superior intellectual functioning as having borderline deficient or deficient intelligence. In line with Willcock et al.'s [7] findings, DAP:QSS scores appeared to be of little use as indicators of children's intellectual functioning.

In sum, the contradictory increase in DAP:QSS mean scores as a function of age, the modest correlations with Raven's Progressive Matrices, and the lack of significant associations between drawings and grades all clearly reveal the weaknesses in both construct validity and concurrent validity of the DAP:QSS as a measure of general intellectual ability. Moreover, in the light of its inaccuracy in intelligence classification, to the key question of whether it is possible to use the DAP:QSS to identify children who might be at risk of intellectual difficulties, it is possible to answer that decisions about intelligence functioning should never be based upon scores for drawings [5, 15].

Altogether, the study's results add further support to the research indicating that the human figure drawings-even in the most up-to-date version (e.g., DAP:IQ) - may not be a valid measure of cognitive ability [50, 51].

Several limitations in the study warrant attention. First, the sample, despite its size, was recruited out of convenience and did not include children with mental disabilities. Moreover, the construct validity was examined by analyzing the increase in mean scores as a function of age; therefore, aspects of the construct validity of the DAP:QSS remain to be evaluated, especially discriminant validity. Data of the drawings was interpreted with reference to the original score norms of Naglieri's drawings. It should be taken into account that DAP:QSS was normed in 1988, and adjustment for the Flynn effects-although observed in human figure drawings [52]—is actually lacking in Naglieri's drawings and therefore would have been needed for this study. In addition, the assessment of academic achievement was based on grades only, not standardized measures, and performed not as an output in specific subject areas but as a global achievement that might not accurately reflect general cognitive abilities at the individual level. 


\section{Summary}

In this study we aimed at assessing the psychometric properties of the DAP:QSS. Although our results produced encouraging evidence of the reliability of Naglieri's drawings, support for the validity of the drawings as a measure of nonverbal intelligence was rather weak. The DAP:QSS also appeared to be an inaccurate measure of academic performance and ineffective in screening for intellectual ability.

In line with the criticism of psychometric qualities shown by previous and more recent versions of DAP, DAP:QSS consistently failed to produce a psychometrically sound assessment for children's intellectual functioning. Taken together, this evidence indicates that the utility of human figure drawings as a measure of intelligence is particularly poor, leading to the conclusion that practitioners should not rely on human figure drawing tests as a projective measure of intelligence.

Acknowledgements The authors thank the director and the teachers of all the schools, parents and children who agreed to take part in the investigation.

Author Contributions The authors contributed equally to the study, AT designed the study, analysed the data and wrote the manuscript. AC, MG, ADL, and CC collected data and contributed to the data analyses and to the manuscript.

Funding Open access funding provided by Università degli Studi della Campania Luigi Vanvitelli within the CRUI-CARE Agreement. The research leading to these results received funding from the project, DiabEaT1, which received funding from University of Campania "Luigi Vanvitelli" through the programme V:ALERE 2019, funded with D.R. 906 del 4/10/2019, prot. n. 157264, October 172019.

Data Availability No prior dissemination of the data and narrative interpretations of the data/research appearing in the manuscript (e.g., data presented at a conference or meeting, posted on a listserv, shared on a website, including academic social networks like ResearchGate, etc.) occurred.

\section{Compliance with Ethical Standards}

Conflict of interest The authors declare that they have no conflict of interest.

Ethical Approval This study was performed in accordance with the ethical standards as laid down in the 1964 Declaration of Helsinki and its later amendments or comparable ethical standards.

Open Access This article is licensed under a Creative Commons Attribution 4.0 International License, which permits use, sharing, adaptation, distribution and reproduction in any medium or format, as long as you give appropriate credit to the original author(s) and the source, provide a link to the Creative Commons licence, and indicate if changes were made. The images or other third party material in this article are included in the article's Creative Commons licence, unless indicated otherwise in a credit line to the material. If material is not included in the article's Creative Commons licence and your intended use is not permitted by statutory regulation or exceeds the permitted use, you will need to obtain permission directly from the copyright holder. To view a copy of this licence, visit http://creativecommons.org/licenses/by/4.0/.

\section{References}

1. Lilienfeld SO, Wood JM, Garb HN (2000) The scientific status of projective techniques. Psychol Sci Public Interest 1(2):27-66

2. Mathijssen AS, Feltzer MJ, Hoogeveen L (2018) Identifying highly gifted children by analyzing human figure drawings: a literature review and a theoretical framework. Psychol Test Assess Model 60(4):493-515

3. Motta RW, Little SG, Tobin MI (1993) The use and abuse of human figure drawings. Sch Psychol Q 8(3):162

4. Naglieri JA (1988) Draw-a-Person: a Quantitative Scoring System. The Psychological Corporation, San Antonio

5. Abell SC, Wood W, Liebman SJ (2001) Children's human figure drawings as measures of intelligence: the comparative validity of three scoring systems. J Psychoeduc Assess 19(3):204-215

6. Gross J, Hayne H (1999) Drawing facilitates children's verbal reports after long delays. J Exp Psychol Appl 5:265-283

7. Willcock E, Imuta K, Hayne H (2011) Children's human figure drawings do not measure intellectual ability. J Exp Child Psychol 110(3):444-452

8. Naglieri JA (1993) Human figure drawings in perspective. Sch Psychol Q 8(3):170-176

9. Wisniewski JJ, Naglieri JA (1989) Validity of the draw a person: a quantitative scoring system with the WISC-R. J Exp Child Psychol 7(4):346-351

10. Haddad FA, Juliano JM (1991) Relations among scores on Matrix Analogies Test, Draw-a-Person, and the Iowa Tests of Basic Skills for low socioeconomic children. Psychol Rep 69(1):299-302

11. Saklofske DH, Braun SM (1992) A psychometric study of the Draw-a-Person: a Quantitative Scoring System. Can J Sch Psychol $8(1): 111-115$

12. Saklofske DH, Tamaoka K, Hildebrand D (1996) An examination of Japanese Children's performance on the Draw a Person: a Quantitative Scoring System. Psychologia 39:17-23

13. Prewett PN, Bardos AN, Naglieri JA (1988) Use of the Matrix Analogies Test-Short Form and the Draw a Person: a quantitative scoring system with learning-disabled and normal students. J Psychoeduc Assess 6(4):347-353

14. Bardos AN, Softas BC, Petrogiannisc K (1989) Comparison of the Goodenough-Harris and Naglieri's Draw-a-Person scoring systems for Greek children. Sch Psychol Int 10(3):205-209

15. Troncone A (2014) Problems of "Draw-a-Person: a Quantitative Scoring System" (DAP: QSS) as a measure of intelligence. Psychol Rep 115(2):485-498

16. Nasvytienë D (2007) The analysis of psychometric properties of human figure drawings' test. Psichologija 36:61-73

17. Prewett PN, Bardos AN, Naglieri JA (1989) Assessment of mentally retarded children with the Matrix Analogies Test-Short Form, Draw A Person: a quantitative scoring system, and the Kaufman Test of Educational Achievement. Psychol Sch 26(3):254-260

18. Gresham FM (1993) "What's wrong in this picture?": Response to Motta et al.'s review of human figure drawings. Sch Psychol Q $8(3): 182-186$

19. Scott LH (1981) Measuring intelligence with the GoodenoughHarris drawing test. Psychol Bull 89(3):483

20. Lassiter KS, Bardos AN (1995) The relationship between young children's academic achievement and measures of intelligence. Psychol Sch 32(3):170-177 
21. Lange-Küttner C, Küttner E, Chromekova M (2014) Deterioration and recovery of DAP IQ scores in the repeated assessment of the Naglieri Draw-A-Person (DAP) test in 6-to 12-year-old children. Psychol Assess 26(1):297

22. Mathijssen AS, Feltzer MJ, Hoogeveen L (2016) Identifying highly gifted children by analyzing human figure drawings: an explorative study. Talent Dev Excell 8:41-53

23. Carmody DP, Crossman AM (2011) Artful liars: malingering on the Draw-a-Person task. Open Criminol J 4:1-9

24. Bandeira DR, Costa A, Arteche A (2012) The Flynn effect in Brazil: examining generational changes in the Draw-a-Person and in the Raven's Coloured Progressive Matrices. Rev Latinoam Psicol 44(3):9-18

25. Lange-Küttner C (2011) Sex differences in visual realism in drawings of animate and inanimate objects. Percept Mot Skills 113(2):439-453

26. Gentle M, Powell MB, Sharman SJ (2014) Mental context reinstatement or drawing: which better enhances children's recall of witnessed events and protects against suggestive questions? Aust J Psychol 66(3):158-167

27. Schepers S, Deković M, Feltzer M, de Kleine M, van Baar A (2012) Drawings of very preterm-born children at 5 years of age: a first impression of cognitive and motor development? Eur J Pediatr 171(1):43-50

28. Kamphaus RW, Pleiss KL (1991) Draw-a-Person techniques: tests in search of a construct. J Sch Psychol 29(4):395-401

29. Anastasi A (1988) Psychological testing, 6th edn. Macmillan, New York

30. Von Stumm S, Plomin R (2015) Socioeconomic status and the growth of intelligence from infancy through adolescence. Intelligence 48:30-36

31. Caro DH, McDonald JT, Willms JD (2009) Socio-economic status and academic achievement trajectories from childhood to adolescence. Can J Educ 32(3):558-590

32. Sirin SR (2005) Socioeconomic status and academic achievement: a meta-analytic review of research. Rev Educ Res 75:417-453

33. Belacchi C, Scalisi TG, Cannoni E, Cornoldi C (2008) CPM Coloured Progressive Matrices: standardizzazione Italiana. Giunti OS, Firenze

34. Picone L, Orsini A, Pezzuti L (2017) Raven's Standard Progressive Matrices: contribution to Italian standardization for subjects between ages 6 and 18. BPA Appl Psychol Bull (Bollettino di Psicologia Applicata) 65:70-81

35. Mackintosh NJ (1996) Sex differences and IQ. J Biosoc Sci 28:558-571

36. Raven JC (1947) Coloured progressive matrices. Lewis, London

37. Raven J, Raven J (2003) Raven Progressive Matrices. In: Steve R, McCallum RS (eds) Handbook of nonverbal assessment. Kluwer, New York, pp 223-237

38. Raven JC (1984) Progressive Matrici Colore Serie A, Ab, B, Manuale di istruzioni (Italian version Raven Progressive Matrices). Organizzazioni Speciali, Florence
39. Raven JC (1958) Raven's Progressive Matrices. University Press, London

40. Costenbader V, Ngari SM (2001) A Kenya standardization of the Raven's coloured progressive matrices. Sch Psychol Int 22:258-268

41. Cotton SM, Kiely PM, Crewther DP, Thomson B, Laycock R, Crewther SG (2005) A normative and reliability study for the Raven's Coloured Progressive Matrices for primary school aged children from Victoria, Australia. Personal Individ Differ 39(3):647-659

42. Kazlauskaite V, Lynn R (2002) Two-year test-retest reliability of the colored progressive matrices. Percept Mot Skills 95(2):354-354

43. MacAvoy J, Orr S, Sidles C (1993) The Raven Matrices and Navajo children: normative characteristics and culture fair application to issues of intelligence, giftedness, and academic proficiency. $\mathbf{J}$ Am Indian Educ 33(1):32-43

44. Rushton JP, Skuy M (2000) Performance on Raven's Matrices by African and White university students in South Africa. Intelligence 28(4):251-265

45. Barratt W (2006) The Barratt simplified measure of social status (BSMSS): measuring SES. Unpublished manuscript, Indiana State University. Retrieved from https://wbarratt.indstate.edu/socialclas s/Barratt_Simplifed_Measure_of_Social_Status.pdf

46. Hollingshead AB (1975) Four Factor Index of Social Status. Unpublished working paper, Department of Sociology, Yale University, New Haven, CT

47. Kahill S (1984) Human figure drawing in adults: an update of the empirical evidence, 1967-1982. Can Psychol 25(4):269

48. Chee KH, Pino NW, Smith WL (2005) Gender differences in the academic ethic and academic achievement. Coll Stud J 39:604-618

49. Richardson M, Abraham C (2009) Conscientiousness and achievement motivation predict performance. Eur J Personal 23:589-605

50. Imuta K, Scarf D, Pharo H, Hayne H (2013) Drawing a close to the use of human figure drawings as a projective measure of intelligence. PloS One 8(3):e58991

51. Rehrig G, Stromswold K (2018) What does the dap: IQ measure?: Drawing comparisons between drawing performance and developmental assessments. J Genet Psychol 179(1):9-18

52. Genovese JE (2018) Evidence of a Flynn effect in children's human figure drawings (1902-1968). J Genet Psychol 179(4):176-182

Publisher's Note Springer Nature remains neutral with regard to jurisdictional claims in published maps and institutional affiliations. 